\title{
Video Article \\ A Pressure Injection System for Investigating the Neuropharmacology of Information Processing in Awake Behaving Macaque Monkey Cortex
}

\author{
Vera K. Veith ${ }^{1}$, Cliodhna Quigley ${ }^{1}$, Stefan Treue ${ }^{1,2}$ \\ ${ }^{1}$ Cognitive Neuroscience Laboratory, German Primate Center \\ ${ }^{2}$ Faculty of Biology and Psychology, Goettingen University \\ Correspondence to: Vera K. Veith at VVeith@dpz.eu
}

URL: https://www.jove.com/video/53724

DOI: doi:10.3791/53724

Keywords: Behavior, Issue 109, visual spatial attention, macaque monkey, pressure injection, scopolamine, extracellular recording, electrophysiology, microinjection, awake behaving animal, drug delivery

Date Published: 3/14/2016

Citation: Veith, V.K., Quigley, C., Treue, S. A Pressure Injection System for Investigating the Neuropharmacology of Information Processing in Awake Behaving Macaque Monkey Cortex. J. Vis. Exp. (109), e53724, doi:10.3791/53724 (2016).

\section{Abstract}

The top-down modulation of feed-forward cortical information processing is functionally important for many cognitive processes, including the modulation of sensory information processing by attention. However, little is known about which neurotransmitter systems are involved in such modulations. A practical way to address this question is to combine single-cell recording with local and temporary neuropharmacological manipulation in a suitable animal model. Here we demonstrate a technique combining acute single-cell recordings with the injection of neuropharmacological agents in the direct vicinity of the recording electrode. The video shows the preparation of the pressure injection/recording system, including preparation of the substance to be injected. We show a rhesus monkey performing a visual attention task and the procedure of single-unit recording with block-wise pharmacological manipulations.

\section{Video Link}

The video component of this article can be found at https://www.jove.com/video/53724/

\section{Introduction}

In cortical and subcortical areas, neuronal activity is affected by various neuromodulators, for example acetylcholine ${ }^{1}$. These modulatory effects on neuronal responses have been reported in in vitro studies ${ }^{2}$, as well as in electrophysiological recordings from anesthetized animals ${ }^{3}$ and systemic pharmacological manipulations in humans ${ }^{4}$. Nevertheless, the exact role of different neuromodulators and the involvement of various receptor subtypes are largely unknown. To measure the effects of specific neuromodulators on the activity of single neurons, it is desirable to induce a temporary neuromodulator change as close as possible to the recording electrode. Furthermore, it is important that those manipulations are done in awake animals, as cognitive functions are only present in the absence of anesthesia. Additionally, anesthesia interacts with cholinergic and GABAergic systems ${ }^{5,6}$ and can lead to changes in neural activity ${ }^{3}$.

Within the last decades, two main methods of local drug delivery have been developed and refined: iontophoresis and pressure injection. In both methods drugs are delivered through micropipettes made of either glass or steel. With iontophoresis, an electrical current regulates the release of the $\mathrm{drug}^{7}$. Additionally, there is a significant contribution of electro-osmosis to the total amount of ejected molecules ${ }^{8}$, correlating with the tip diameter $^{9}$ of the micropipette as well as with the concentration ${ }^{8}$ of the substance used. Iontophoresis is a powerful tool to quickly and precisely manipulate small volumes of nervous tissue. For iontophoretic injections, multi-barrel micropipettes are usually used ${ }^{10}$, with one acting as a recording device while the other positions serve as delivery pipettes. A limitation of this method is that only charged molecules can be used, severely limiting the selection of drugs.

Pressure injection uses either air compression or mechanical pressure to eject a substance from a micropipette. Using this method any soluble substance, charged or uncharged, can be used, including large molecules. The method of pressure injection was first described by Reyniers in 1933 and further refined in the 1950 s (see Lalley ${ }^{11}$ for a review). In the 1980 s the method was further refined to allow delivery of amounts in the nanoliter range (mainly lidocain ${ }^{12}$ ) to a defined brain area ${ }^{13}$ while simultaneously performing single-cell recording. The ejected volume was usually monitored by observing the movement of a marker, such as the meniscus in the upper part of the pipette ${ }^{13}$. Pressure injection was first used in the 1990s in awake animals, both extracellularly ${ }^{14}$ and intracellularly ${ }^{15,16}$. Based on the cumulative expertise gained in these studies it is now possible to reliably record from different brain structures in combination with pharmacological manipulation (see ${ }^{17}$ for a comparison of recent pressure injection systems).

An enduring open issue for both drug delivery methods is the difficulty in determining the precise volume injected. This is an even bigger challenge for experiments with awake, behaving rhesus monkeys where the animal performs the experimental task in a separate room. This can be alleviated by the use of a software-controlled system instead of relying on a visual marker to continuously monitor an injection. 
The system described here is an extension of a well-established electrophysiological recording system (Mini Matrix System) and combines an injection pipette with multiple parallel-oriented recording electrodes at defined distances in a customizable arrangement. Pharmacological manipulation of the tissue near the recording electrode is possible using only a small amount of substance, ensuring a fast recovery and allowing multiple blocks of injection and control/recovery within the limited time window offered by the behavioral task of the animal.

\section{Protocol}

Animal care and all experimental procedures were conducted in accordance with German laws governing animal care and approved by the district government of Braunschweig, Lower Saxony, Germany.

Note: As the experiment is performed in vivo, it is crucial to maintain the highest possible hygiene standards. Whenever possible, work under sterile conditions.

\section{Preparing the Injection/Recording System}

1. Sterilize the tube that connects the micropipette with the syringe. Use the shortest length of tube possible in the experimental set-up between injection pump and electrophysiological recording system.

2. Clean the guide tubes of the recording system using cleaning wires. Dip them in sterile silicon oil and feed them through the individual guide tubes several times.

3. Insert the quartz glass micropipette into one guide tube of the recording system. See Figure 1A

4. After fixing the micropipette in the recording system, attach the sterile tube to the metal pin of the micropipette. Take care; although the micropipette is fixed in the system it can easily break when attaching the tube. Use two sterile tweezers to apply equal pressure on pin and tube.

5. Use liquid super glue to seal the junction between tube and micropipette. Wait at least $3 \mathrm{hr}$ for the glue to harden before filling the micropipette with liquid.

6. Insert microelectrodes (e.g., quartz glass insulated platinum tungsten) into the other positions of the recording system before or after micropipette insertion.

\section{Preparing the Substance}

1. Sterilize $1.5 \mathrm{ml}$ microcentrifuge tubes for later storage of injection solutions using an autoclave or other reliable procedure.

2. Weigh the corresponding substance scopolamine hydrochloride to prepare $5 \mathrm{ml}$ of a 0.1 molar solution. Dissolve in sterile saline $(0.9 \% \mathrm{NaCl})$.

3. Under sterile conditions in a fume hood, filter the solution using a syringe filter with sufficiently large pore diameter, e.g., $0.2 \mu \mathrm{m}$.

4. Under the fume hood, aliquot the solution into volumes sufficient for a single experiment, e.g., for scopolamine, $500 \mu \mathrm{l}$ in sterile $1.5 \mathrm{ml}$ microcentrifuge tube. Use dark tubes to protect the substance from light; alternatively, wrap tubes in aluminum foil. For scopolamine, store the solution for up to 14 days at $4{ }^{\circ} \mathrm{C}$.

\section{Daily Preparation of the Injection/Recording System}

Note: When mounted in the recording system, the electrodes and micropipette are stored in an enzyme solution (Tergazyme, $1 \%$ solution with deionized water) between recordings. The following steps must be performed before every recording.

1. Collect a tube of the substance to be injected. Allow it to reach RT if refrigerated.

2. Remove the injection/recording system from the enzyme solution and rinse electrodes and micropipette with deionized water to clean completely of enzyme solution.

3. Remove the front cover of the recording system in order to visually check the seal between micropipette and tube.

4. Apply sterile silicon oil to the guide tube gap (see Figure 1A) and tips of electrodes and micropipette in order to lubricate the system for smooth movement.

5. Check tips of electrodes and micropipette using a microscope to ensure they are intact. Align the electrodes and the micropipette under the microscope so that they extend out of the guide tubes with the same length. Drive them into the guide tubes, stopping as soon as they are no longer visible. This is defined as electrode position zero. Set the depth of the electrodes and micropipette to 0 in the software.

6. Fill a sterile syringe with sterile saline and insert the needle into the tube, taking care not to pierce the wall of the tube. Drive the micropipette out of the guide tube for visual control of substance flow.

7. Flush at least $2 \mathrm{ml}$ sterile saline through the tube and micropipette to ensure no air remains in the syringe or in the tube. Do not apply too much pressure to the plunger of the syringe. Ensure the junction between tube and micropipette is sealed. If leakage is visible, re-glue the junction (see step 1.5) and postpone recording.

8. Fill a new sterile syringe with the solution to be injected and exchange it with the barrel of the saline syringe, i.e., keep the needle of the saline-filled syringe in the tube. Make sure that no air is transferred into the system. This is best achieved by filling the needle hub with saline after removing the saline-filled barrel.

9. Flush the system with $250 \mu \mathrm{l}$ of the solution to be injected, in order to completely remove the saline from the tube.

10. Using the motor control software, retract electrodes and micropipette into the guidetubesto a depth of at least $-500 \mu \mathrm{m}$.

11. Lower the guide tube ring (Figure 1A) to the bottom of the guide tubes to maintain their fixed relative position.

12. Clean the base of the system with ethanol, in particular where it will touch the monkey's recording chamber.

13. Close the recording system by replacing the front cover and tightening the screws. 


\section{Validation of the Injection System}

Note: Although the company calibrates the system, it is recommended to validate the ejected volumes with the materials used in the experimental set-up (tubes, syringes etc.).

1. Prepare the system as described in step 3, keeping electrodes and micropipette extended out of the guide tubes. A depth of at least 7,000 $\mu \mathrm{m}$ is recommended to avoid loss of measurement volume due to adhesion along the outer surface of the micropipette and electrodes.

2. Place the recording system in the position it will be used in during the experiment and put the syringe into the microinjection pump. Fix the syringe in place using the rubber band and adjustable grip (see Figure 1A). Slide the movable part of the pump until it is firmly in place behind the plunger of the syringe.

3. Using the software-controlled motor unit, eject a volume large enough to be measured precisely, e.g., 1,000 nl. It is preferable to use one single step to eject the total volume in order to avoid effects of capillary action along the micropipette surface. Very low velocities (1 $\mathrm{nl} / \mathrm{s})$ can also lead to this effect during the validation procedure.

4. Collect the total volume in a container placed under the micropipette, or carefully collect the ejected drop directly from the tip of the micropipette. Estimate the ejected volume using a pipette or by weighing with a precision scale.

5. Repeat the procedure several times to confirm measurements.

\section{Acute Recordings}

1. Set the $x-y$ position of the recording system. This defines the point at which the guide tubes reach the dura mater within the chronically implanted recording chamber. Make sure the guide tubes are retracted completely (guide tube $z$ position 0 ).

2. Bring the recording system into position and place the syringe in the microinjection pump. Fix the syringe in place using the rubber band and adjustable grip (see Figure 1A). Slide the movable part of the pump until it is firmly in place behind the plunger of the syringe. If a drop of substance is visible at the tip of the guide tubes, carefully remove it using a sterile cotton bud.

3. Prepare the animal for recording according to the laboratory's procedure (see ${ }^{18}$ for example guidelines).

4. Securely mount the recording system on the recording chamber of the monkey.

5. Slowly manually lower the guide tubes into the recording chamber until the dura is reached, then drive the electrodes using the motor control software.

6. As it is not possible to measure impedance of the micropipette, first drive with electrodes and check their impedances regularly at different depths. After a penetration of the dura is successfully performed without damaging the electrodes, advance the micropipette.

7. Drive the electrodes and the micropipette to the target electrode depth at which the brain area of interest is expected to be found. Slowly advance the electrode until it is close enough to record the activity of a single unit, as evidenced by a good signal-to-noise ratio in the recorded signal. Importantly, position the recording electrode and the micropipette at the same depth to ensure the minimum distance between electrode and micropipette.

1. If possible, keep the electrodes and micropipette at this depth for the entire recording. However, if the only way to maintain signal quality of the recorded cell is to move the electrodes, then drive the electrodes and the micropipette simultaneously to maintain the distance between them.

\section{Spatial Attention Task}

1. In a series of trials, present two moving dot patterns on the screen, one positioned within the receptive field of the recorded neuron and the other outside of it, together with a centrally presented fixation point that the animal has to foveate throughout each trial ${ }^{19,20}$

Note: The monkey is trained to respond to a direction change in the cued dot pattern (the target event) while ignoring any direction change in the other dot pattern, and is rewarded with a drop of liquid for every successful completion of a trial ${ }^{19,20}$. As a sensory control condition, the monkey has to report a luminance change of the fixation point while ignoring both moving dot patterns (see Figure 2 for a more detailed description of the task).

\section{Pharmacological Manipulation While Recording}

Note: While the monkey is performing the task, inject the substance in a block-wise manner. Three consecutive blocks are defined: control, which acts as a baseline; injection, during which a substance is ejected; and recovery, during which the cells targeted by the injection return to baseline.

1. During an injection block, inject a predefined amount of the substance at regular intervals e.g., $2 \mathrm{nl}$ every minute at a rate of $2 \mathrm{nl} / \mathrm{s}$. For this example, use scopolamine hydrochloride. The injection process is controlled using software which provides various options. For example, use the step function to define injection volume, and press the injection button every minute according to the clock of the recording software. Note: The exact duration of the injection block is substance and experiment dependent, e.g., for scopolamine use $2 \mathrm{nl}$ injections each minute for $10 \mathrm{~min}(20 \mathrm{nl}$ in total). It is preferable not to advance the electrodes and micropipette during the injection block.

2. Note the time and the trial during which the substance is injected, the depth of the electrodes and micropipette, as well as the amount of ejected substance.

3. Follow the injection block with a recovery block, in which no substance is injected. The duration of the recovery block is substance specific and needs to be defined in pre-tests. Monitor and maintain the recording quality of the selected single units until the end of the recovery block.

4. Repeat the three blocks for as long as the recording quality and motivation of the monkey allow. 


\section{Post Recording Procedures}

1. After data recording, retract electrodes and micropipette into the guide tubes and then manually retract the guide tubes. Remove the recording system from the recording chamber of the monkey. Release the syringe from the injection pump and transfer the system to the preparation area for cleaning.

2. Handle the animal (including cleaning of the recording chamber ${ }^{18}$ ) according to the standard procedures of the laboratory and return it to the housing facility.

3. Rinse the outside of the guide tubes with hydrogen peroxide (3\%) and then with deionized water. Drive electrodes and micropipette out of the guide tubes, rinse with hydrogen peroxide and then deionized water.

4. Exchange the barrel of the syringe with a barrel of a syringe filled with sterile saline, keeping the needle in the tube. Flush the tube and the micropipette with 1-2 ml of saline. After flushing, remove the barrel and fill it with air. Reinsert the barrel into the needle and dry the tube and the micropipette from the inside by gently pushing air through.

5. Store the guide tubes, extended electrodes and micropipette immersed in the enzyme solution to avoid drying as well as to ensure the breakdown of organic material.

\section{Representative Results}

Figure 2 depicts the spatial attention task the monkey performed while the injection process was conducted. The monkey was trained to attend to either the stimulus located within the receptive field of the recorded neuron (attend-in), the stimulus located outside of the receptive field (attend-out) or the fixation point (attend-fix). These conditions allow a comparison of neuronal activity in different attentional states.

Figure 3 shows a peri-stimulus time histogram of a sample neuron in an experiment using scopolamine, a muscarinic cholinergic antagonist. The plot demonstrates the response suppression during scopolamine injection versus no injection, when a pattern moving in the cell's preferred direction is presented inside the neuron's receptive field and is attended by the animal. The two first peaks represent the neuron's response to the on- and offset of the spatial cue, which appears inside its receptive field. This is followed by the response to the moving pattern which appears on the screen $500 \mathrm{~ms}$ after cue onset. The gray shaded area depicts the analysis period used to calculate the average firing rate for every trial. The green area highlights the suppressive influence of scopolamine injection on the cell's firing rate. The dark green region shows the suppression within the analysis period.

Figure 4A shows the effect of scopolamine on the average firing rate of the sample neuron in each of the three attentional conditions. The neuron's firing rate for the two spatial attention conditions (attention inside or outside of the receptive field of the recording neuron) as well as for the sensory condition (attention at fixation point) dropped shortly after the first injection of the injection block (grey shaded area) and during the recovery block increased after a delay to the same level as before the injection.

Figure 4B shows a control recording from a second sample neuron in which saline $(0.9 \% \mathrm{NaCl})$ was injected, using the same protocol as for the scopolamine injection. During the injection block no change in the neuron's firing rate was observed compared to the control block.

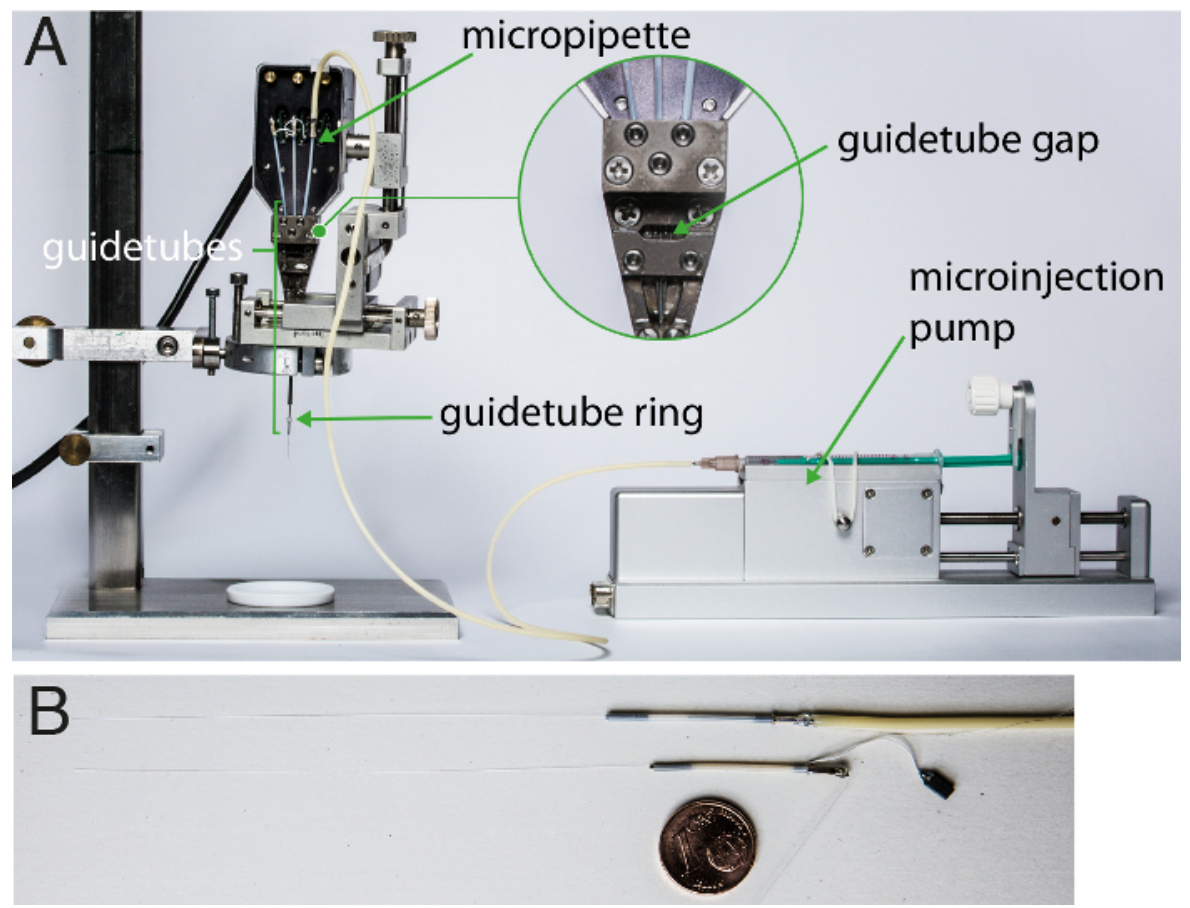

Figure 1. Set-up used for pharmacological manipulation while recording. (A) Depicts the microinjection pump and the electrophysiological recording system equipped with electrodes and micropipette. The guidetube gap, into which silicon oil is inserted to lubricate the electrodes and micropipette, is shown enlarged. (B) Displays an example micropipette (above) and recording electrode (below). For size comparison, a euro cent (diameter: $16 \mathrm{~mm}$ ) is placed underneath. Please click here to view a larger version of this figure. 


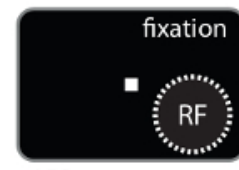

$150 \mathrm{~ms}$

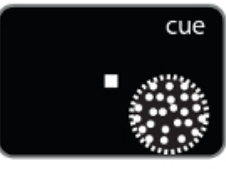

$150 \mathrm{~ms}$

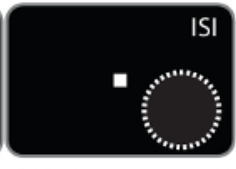

$350 \mathrm{~ms}$

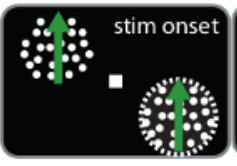

200-2500ms

Figure 2. Task design to guide spatial attention. Monkeys were trained to detect a motion direction change in the cued dot pattern. The cue was either placed within the neuron's receptive field (attend-in), as shown in the figure, or outside of it (attend-out). As a sensory control, the monkey was trained to detect a luminance change of the fixation point (attend-fix). Please click here to view a larger version of this figure.

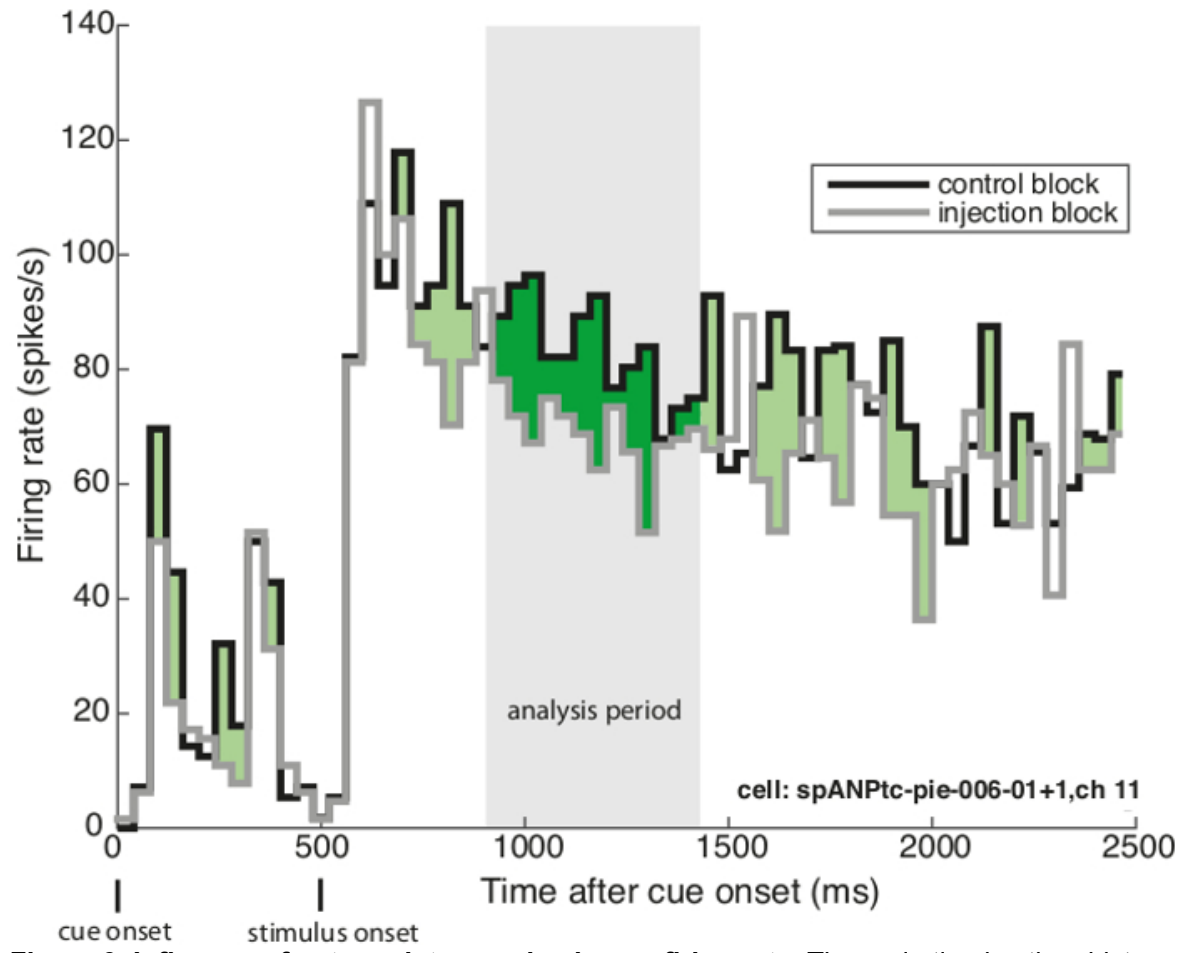

Figure 3. Influence of antagonist scopolamine on firing rate. The peri-stimulus time histogram for a sample neuron is shown for the attendin condition (attention inside the receptive field of the recorded neuron) during the injection block and during the control block. The $x$-axis depicts the time in milliseconds after cue onset and the $y$-axis shows the firing rate in spikes/sec. The grey area depicts the analysis period (300-800 ms after stimulus onset) used to calculate the trial-averaged firing rate. The green shaded area shows the suppression in firing rate across the two conditions. The dark green color highlights the suppression within the analysis period. Please click here to view a larger version of this figure. 

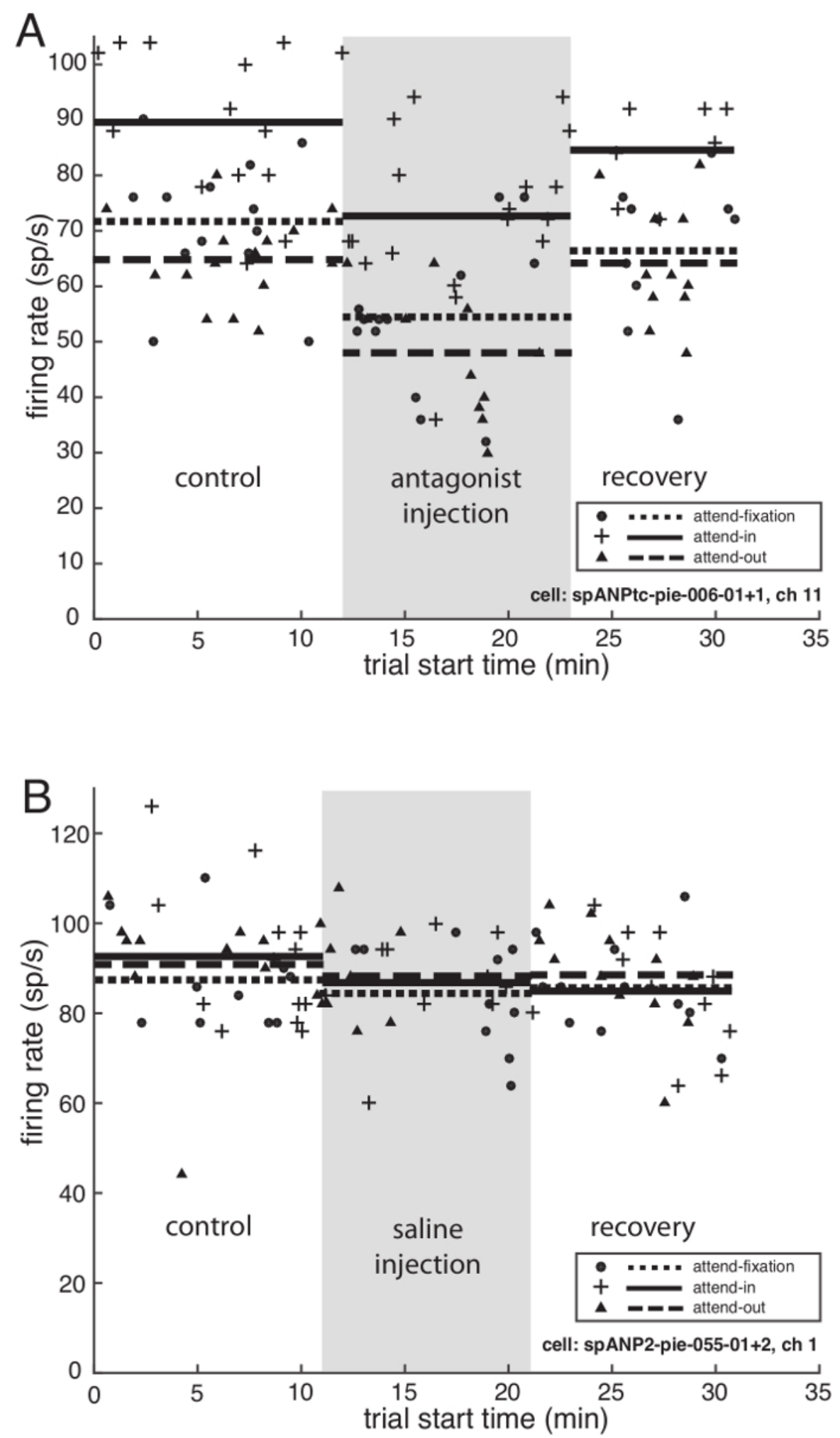

Figure 4. Effect of scopolamine and saline on firing rate. (A) Antagonist scopolamine injection. The trial-averaged firing rate of the sample cell from Figure 3 over the course of the experiment is shown for the preferred stimulus for all three attentional conditions. The $x$-axis depicts the trial start time in minutes and the $y$-axis shows the unit's firing rate in spikes per seconds. Symbols ( + attend-in, $\bullet$ attend-fix, $\Delta$ attend-out) represent the neuron's firing rate within the analysis period in every successfully performed trial, and horizontal lines (solid line: attend-in, dotted line: attend-fix, dashed line: attend-out) show average firing rate for the three different experimental blocks (control, injection, recovery). The grey shaded area shows the injection block, beginning with the first injection and ending $1 \mathrm{~min}$ after the last injection. During the injection block $2 \mathrm{~nL}$ of 0.1 molar scopolamine were injected every minute with an injection velocity of $2 \mathrm{nl} / \mathrm{s}$. (B) Saline injection. The firing rate of a sample cell over the 
course of the control experiment is shown for the preferred stimulus for all three attentional conditions. Grey shaded area visualizes the block of saline injection. Please click here to view a larger version of this figure.

\section{Discussion}

Here we have illustrated in detail how to perform reliable and precise injections and high quality single-cell recordings with an "off-the-shelf" pressure injection system. While this method of drug delivery has previously been used in behaving monkeys (reviewed in ${ }^{17}$ ), the system presented here has advantages, reviewed below.

As illustrated in Figure 4A, the system described here can provide stable measurement of single neuron activity with and without pharmacological injections in the direct vicinity of the recording site. As shown in Figure 4B, the injection of a control substance, saline, did not lead to a change in firing rate. This control demonstrates that the injection process itself has no measurable influence on the firing properties of the recorded neurons.

The spatial configuration of neuron, recording electrode, and micropipette is of crucial importance in these experiments. Although a precise measurement of their relative positions in the tissue during recording is not possible, we can consider and control for possible sources of variance. First, during volume injection there is a risk that the neuron of interest may be displaced away from the recording electrode, affecting the stability of recorded signals. For that reason it is prudent to compare the firing rate before and after the injection block to verify signal stability. Second, the guide tube configuration of the recording system defines the distance between electrodes and micropipette (e.g., $305 \mu \mathrm{m}$ in the concentric 3-channel system used in this experiment). As the system provides precise position control for the depth of electrodes and micropipette in the tissue, the distance between them can be minimized by carefully calibrating relative depth before recording (step 3.5), and keeping them at a common depth during recordings.

\section{Potential limitations}

In addition to in-house quality control by the manufacturer, the system needs to be validated under lab conditions, as different brands of tubes, syringes etc. can be used and could lead to differences in ejected volumes. Although the system can be used to inject very small volumes as in the experiment shown here, these are below the minimum volume that can be validated due to practical measurement limits in a normal laboratory environment. However, larger injection volumes can be used to infer the relation between the software-defined volume and the volume ejected by the hardware. If transparent tubes are used, an additional visual check of the injection process is possible by measuring the displacement of a visual marker.

Inserting the micropipette into the system is more demanding than electrode insertion, as the diameter of the micropipette is slightly larger and the material is more fragile. In addition, joining the tube to the pin of the micropipette is challenging as it entails a high risk of breaking the upper part of the micropipette. However, the lifetime of a successfully loaded micropipette is several months, even with daily use.

In practice, we have not yet encountered a blockage in the injection system during post-recording cleaning of the system. Nevertheless, no "online" check is possible, and there is a risk that a physical blockage (such as tissue at the micropipette tip) might prevent substance injection. It might therefore be advisable to analyze the data conservatively, such as including only those cells in further analysis that show significant changes in firing rates between control and injection blocks of the experiment.

Despite their small diameter, microelectrodes and pipettes will displace brain tissue and may cause some local tissue damage. This can be minimized by manually positioning the tip of the guide tubes just above the dura mater. The electrodes then penetrate the dura and their intactness is inferred by measuring their impedances online. Afterwards, the micropipette is inserted. When using this approach, regular removal of tissue above the dura is recommended to further reduce the risk of electrode or pipette breakage.

\section{Comparison to alternative methods}

The system used here shows clear advantages compared to other pressure injection systems. One strong advantage is the diameter of the micropipette (approximately $100 \mu \mathrm{m}$ ), which is half the size of other available probes ${ }^{17}$ and therefore minimizes neural tissue damage. In contrast to previous designs, the current system employs spatially separated recording electrode and micropipette. Although other systems provide a smaller distance between electrode and pipette, the system described here allows independent depth changes of electrodes and pipette, thus permitting variable relative distances within a recording session. Importantly, no compromise regarding recording quality needs to be made, as the injection system is an extension of an established recording device. While only one micropipette and thus one substance is used in this protocol, it is possible to inject several substances within one experimental procedure. To achieve this, several micropipettes can be threaded into separate guide tubes and connected to syringes mounted in individual injection pumps. Finally, controlling the system is easy, as only one computer program is needed to advance the electrodes and micropipette, and to perform the pressure injection during the experiment.

Comparing pressure injection to iontophoresis, there are relative advantages and disadvantages. For example, pressure injection requires greater volumes to be introduced into the tissue than iontophoresis, thus increasing the risk of neuronal displacement. The current protocol used volumes in the $\mathrm{nL}$ range, and we rarely experienced noticeable changes in a recorded cell's signal quality. The system also allows larger volumes to be injected, which is potentially useful for behavioral manipulations but could impact stability of neuronal recording. A clear advantage of pressure injection over iontophoresis is the larger variety of useable substances as there is no requirement to use charged substances. However, pH values should be checked and compared between experimental and control substances (e.g., saline).

The question might arise why to use the long-established method of pressure injection instead of newer techniques such as optogenetics for manipulating neural activity. Although well established in rodents, optogenetics is not yet reliably established in rhesus monkeys. In particular, it does not yet allow the local manipulation of cells selective for a particular neurotransmitter type. In the longer run, we see great potential for the combination of the advantages of pharmacological manipulations with the advantages of optogentic manipulations in elucidating the neural basis of cognitive functions. 
Here we have shown how pressure injection can be used to pharmacologically manipulate a locally restricted area in the brain of awake, behaving rhesus monkeys. We hope that this method inspires other scientists to investigate neuromodulatory contributions to the dynamics of neuronal activity.

\section{Disclosures}

The authors have nothing to disclose.

\section{Acknowledgements}

This work was supported by grants of the Deutsche Forschungsgemeinschaft through the Collaborative Research Center 889 "Cellular Mechanisms of Sensory Processing" to S.T. (Project C04). We thank Sina Plümer, Leonore Burchardt, Dirk Prüsse, Klaus Heisig and Ralf Brockhausen for technical and animal-related support and our collaborators in the Stem Cell Unit of the German Primate Center, Dr. Katharina Debowski and Anna Magerhans, for technical assistance in the filtration process.

\section{References}

1. Noudoost, B., Moore, T. The role of neuromodulators in selective attention. Trends Cogn Sci. 15(12), 585-591 (2011).

2. Jochems, A., Reboreda, A., Hasselmo, M., Yoshida, M. Cholinergic receptor activation supports persistent firing in layer III neurons in the medial entorhinal cortex. Behav Brain Res. 254,108-115 (2013).

3. Thiele, A., Herrero, J.L., Distler, C., Hoffmann, K.P. Contribution of cholinergic and GABAergic mechanisms to direction tuning, discriminability, response reliability, and neuronal rate correlations in macaque middle temporal area. J Neurosci. 32(47):16602-16615 (2012).

4. Thienel, R., et al. Muscarinic antagonist effects on executive control of attention. Int J Neuropsychopharmacol. 12(10), 1307-1317 (2009).

5. Anthony, B.L., Dennison, R.L., Aronstam, R.S. Disruption of muscarinic receptor-G protein coupling is a general property of liquid volatile anesthetics. Neurosci Lett. 99(1-2), 191-196 (1989).

6. Yamakura, T., Bertaccini, E., Trudell, J.R., Harris, R.A. Anesthetics and ion channels: molecular models and sites of action. Annu Rev Pharmacol Toxicol. 41, 23-51 (2001).

7. Herr, N.R., Wightman, R.M. Improved techniques for examining rapid dopamine signaling with iontophoresis. Front Biosci. 5, 249-257 (2013).

8. Bevan, P., Bradshaw, C.M., Pun, R.Y., Slater, N.T., Szabadi, E. The relative contribution of iontophoresis and electro-osmosis to the electrophoretic release of noradrenaline from multi barrelled micropipettes [proceedings]. Br J Pharmacol. 67(3), 478-479 (1979).

9. Herr, N.R., Kile, B.M., Carelli, R.M., Wightman, R.M. Electroosmotic flow and its contribution to iontophoretic delivery. Anal Chem. 80, 8635-8641 (2008).

10. Thiele, A., Delicato, L.S., Roberts, M.J., Gieselmann, M.A. A novel electrode-pipette design for simultaneous recording of extracellular spikes and iontophoretic drug application in awake behaving monkeys. J Neurosci Meth. 158(2-4), 207-211 (2006).

11. Lalley, P.M. Microiontophoresis and Pressure Ejection: Modern Techniques in Neuroscience. (eds. Windhorst U., \& Johansson H.) 193-209, (1999).

12. Malpeli, J.G., Schiller, P.H. A method of reversible inactivation of small regions of brain tissue. J Neurosci Meth. 1(2), 145-59 (1979).

13. Malpeli, J.G. Reversible inactivation of subcortical sites by drug injection. J Neurosci Meth. 86(2), 119-28 (1999).

14. Dias, E.C., Segraves, M.A. A pressure system for the microinjection of substances into the brain of awake monkeys. J Neurosci Meth. 72 (1), 43-47 (1997).

15. Szente, M.B., Baranyi, A., Woody, C.D. Effects of protein kinase $\mathrm{C}$ inhibitor H-7on membrane properties and synaptic responses of neocortical neurons of awake cats. Brain Res. 506 (2), 281-286 (1990).

16. Woody, C.D., Bartfai, T., Gruen, E., Nairn, A. Intracellular injection of cGMP-dependent protein kinase results in increased input resistance in neurons of the mammalian motor cortex. Brain Res. 386 (1-2), 379-385 (1986).

17. Noudoost, B., Moore, T. A reliable microinjectrode system for use in behaving monkeys. J Neurosci Meth. 194 (2), $218-23$ (2011).

18. Association of Primate Veterinarians. Cranial Implant Care Guidelines for Nonhuman Primates in Biomedical Research. http:// www.primatevets.org/Content/files/Public/education/Cranial\%20Implant\%20Care\%20Guidelines.pdf (2015).

19. Treue, S., Martinez-Trujillo, J.C. Feature-based attention influences motion processing gain in macaque visual cortex. Nature. 399, 575-579 (1999).

20. Martinez-Trujillo, J.C., Treue, S. Feature-based attention increases the selectivity of population responses in primate visual cortex. Curr Biol. 14 (9), 744-751 (2004). 